\title{
Psychiatric Rehabilitation During COVID-19 Pandemic: Richmond Fellowship Society (I), Bangalore Branch Experience
}

\author{
Seshadri Kalyanasundaram (D) - Lata Hemchand • Thanapal Sivakumar
}

Received: 23 July 2021 / Accepted: 16 December 2021 / Published online: 7 January 2022

(C) The Author(s), under exclusive licence to Springer Nature India Private Limited 2022

\begin{abstract}
Richmond Fellowship Society (India) [RFS(I)] is a "Not for Profit" Non-Governmental Organization working for the psychosocial rehabilitation of persons with chronic mental illness. The RFS(I) Bangalore branch runs a daycare, half-way home, and long-stay facility. This paper describes the RFS(I) Bangalore branch's experience running psychiatric rehabilitation facilities during the first two waves of the COVID-19 pandemic, including managing a COVID-19 outbreak in the residential facilities.
\end{abstract}

Keywords COVID-19 - Psychiatric rehabilitation · Mental illness · Vaccination · India $\cdot$ Low-and-middle income countries

\section{Introduction}

Worldwide, the COVID-19 pandemic has led to uncertainties about its spread, the effectiveness of containment strategies, and the uncertainty of the time it will take for life to return to normalcy [3]. Persons with mental illness are among the most vulnerable and

S. Kalyanasundaram $(\bowtie) \cdot$ L. Hemchand · T. Sivakumar Richmond Fellowship Society (I), Bangalore Branch, Bangalore, Karnataka, India

e-mail: sundarps@gmail.com

T. Sivakumar

Department of Psychiatry, National Institute of Mental Health and Neurosciences (NIMHANS), Bangalore, India least privileged social groups [3]. COVID-19 has worsened healthcare for persons with mental illness but has conversely has accelerated technology adoption to replace disrupted in-person mental health services when possible [5-7]. Rehabilitation is a necessary but oft-neglected part of the comprehensive management plan of persons with mental illness [10]. There are limited reports of ensuring continuity of rehabilitation care from lower and middle-income countries during the pandemic $[4,8,12]$.

\section{Richmond Fellowship Society Bangalore Branch}

Richmond Fellowship Society (India) [RFS(I)] is a "Not for Profit" Non-Governmental Organization (NGO) working for psychosocial rehabilitation of persons with chronic mental illness (referred to as 'clients' from hereon). The Bangalore branch, founded in 1986, has been a pioneer in rehabilitation in India [11]. It offers the following facilities.

(a) Half-way Home-'Asha' for 21 clients (both men and women)

(b) Long-stay Home-'Jyothi' for 17 clients (men and women)

(c) Day Care Centre with Vocational training'Chetana' for 50 clients

All three centers provide treatment and rehabilitation support to clients to re-acquire skills across affected 
domains in a therapeutic environment for personal growth and offer an opportunity to regain self-respect. In addition, individual and family counseling services are provided. The Fellowship also offers respite care on a case-to-case basis.

At the Vocational training center, skills such as Basic training in Computers, offset and screen printing, block-printing, tailoring, and embroidery are taught to the clients. Paper file making, Paper cup making, Book Binding, and Spiral Binding skills are also imparted to the clients. This helps in developing work habits and functions as a sheltered workshop for some of them.

In addition, the clients and residents are engaged in Green Skilling activity, which involves recycling discarded flowers from the nearby temples, marriage halls and other similar places/events. The process involves segregating and cutting, drying petals, grinding dried petals, mixing, refining, and packaging resulting in "Eco-Friendly Holi/Rangoli Colours."

\section{COVID-19 Pandemic in India}

India is the second-worst affected country due to the pandemic [1]. Lockdowns were declared to contain the first and second waves of the pandemic. The nationwide lockdown imposed during the first wave was unprecedented and posed significant challenges in accessing non-emergency healthcare and public mobility. The risk of acquiring the infection, dread of its spread, quarantine of suspected individuals, physical distancing, and a drastic change in routine for people has led to a wave of mental health concerns [9]. India's health inequalities, flaring economic and social disparities, and distinct cultural values made lockdown hard for the poorer and vulnerable sections of society [2]. The restrictions during the lockdown for the second wave were relatively less stringent and permitted better movement.

Unfortunately, rehabilitation is not considered an essential service like healthcare. Unlike daycare services, residential rehabilitation services cannot be closed due to COVID-19. The present paper describes the experience of the RFS (I) Bangalore branch in running rehabilitation facilities during the pandemic. There were 11 professional staff before the onset of the pandemic. Eight of them had done masters in social work (medical \& psychiatry). 2 staff had done MSc in
Psychosocial Rehabilitation and counseling, and; 1 staff had done MSc in Psychology. The staff had 2-6 years of experience working in the facilities and trained in RFS ethos.

\section{Impact on Daycare Facility (Chetana)}

As clients traveled from various locations to avail benefits at Chetana, the management recognized the possibility of transmission of infection. It was decided to temporarily close Chetana during both pandemic waves (15th March 2020 to 13th September 2020 and 28th April 2021 till date), much before the Government declared a lockdown. Most families were supportive of the decision.

Thirty-four clients (20 with mental illness and 14 with intellectual disability) were attending Chetana daycare center before the lockdown. When Chetana was closed, the daily routine of most clients was disrupted. After closure, four professional staff were handling calls and clarifying their doubts. Gradually the number of staff was reduced to 2 . The clients' list was divided among the staff who called the respective clients and caregivers at a mutually convenient time once in 2-3 weeks (depending on the need) to keep up their morale, check medication adherence, help schedule the day, and deal with mental health concerns. The mode of communication was WhatsApp Audio/Video calls depending on the need of the client. Clients were educated about COVID-19 and precautions to be adopted. The staff and family members shared periodic updates in a WhatsApp group. Three families of clients with ID expressed that they are unable to access the video calls due to a lack of resources. For clients with ID, assistance was required-the caregiver would give feedback and later pass on the call to the client.

The staff would encourage the clients to do tasks assigned by them. Some clients' were adamant about following the routine advised, were restless, and sometimes handling them was difficult at home. As per the caregivers' information, there were no signs of relapse in clients with mental illness. Three caregivers of clients with intellectual disabilities reported mood fluctuations and difficulty involving them in household chores. While most clients with mental illness adapted to the situation, clients with intellectual disability were keen to rejoin Chetana. 
Between the two waves, Chetana functioned from 14th September 2020 to 27th April 2021. RFS bus facility was not available, and clients had to make their arrangements for travel. Clients attended Chetana in 2 batches on alternate days till December 2020. Due to limited patronage (only 16/50 clients availed of Chetana services; 10 of them with mental illness), all 16 clients attended daily from January 2021 till 27th April 2021. Many caregivers of clients with intellectual disability had concerns about the ability of the client to follow COVID appropriate behavior and didn't send them to Chetana.

\section{Impact on Residential Services (Asha Half-Way Home and Jyothi Long-Stay Home)}

Most clients were at high risk due to their age and multiple medical comorbidities. RFS adopted adequate precautions at the onset of the first wave of the pandemic. Delivery persons and other visitors, and family members were not allowed inside the residential premises. Therapeutic and recreational off-site visits for clients were suspended. Since the onset of the pandemic, the premises were regularly sanitized twice a day.

The staff ensured social distancing, usage of masks, and hand hygiene among clients. Videos on COVID19 awareness and washing hands helped clients better understand the situation. Staff supervised clients washing their hands regularly. All clients and staff were asked to use a mask, and it was monitored continuously. Temperature and oxygen saturation levels were checked daily for all clients and staff. Physical distancing was maintained by having fewer clients working inside a room for any activity.

Some clients would get worried if they found someone on the premises coughing or sneezing. One client (due to her intense anxiety over getting infected) called to police and corporation administration. Conversely, few others were indifferent to the situation, and staff found it challenging to make them wear a mask. They had to be closely monitored and gently persuaded to follow necessary precautions. Anytime we had the suspicion of possible COVID-19, the concerned person and all people exposed were tested.

As clients watched the news telecast, where most alarming things were discussed, again and again, they were advised to avoid watching such telecast. The staff monitored the content of TV watching to minimize watching excessive negative news.

Some clients started questioning about vegetable vendors and others who were coming on the road to see their wares. The vegetable vendors were asked to wait outside while the staff bought the vegetables. The staff sanitized vegetables delivered. The process was explained to clients which reduced level of anxiety.

The clients were personally reassured and educated about various precautions taken at the facility by the first and second authors associated with running the facility. Reassurance and firmness in handling (when required) were helpful.

The usual recreation programs like snacks out, lunch out, picnic, movie, and Chetana vocational activity were impossible during the lockdown. The clients were understandably distressed that they were not allowed to go for walks, shop, or visit the temple. There was a great deal of annoyance and irritability, not bordering on breakdown, in a couple of the residents. Thus, keeping the clients engaged every day was a great challenge. Efforts were made to prepare special snacks which clients liked so that they did not miss eating out. Films were screened in-house with physical distancing. Special activities like collage making, quiz competitions, group games, music classes, and coloring activities helped clients follow a routine, stay calm and active during the daytime. One client completed her online embroidery course.

It was challenging to ensure continuity of care for all clients, including follow-up consultations and administering injections/electroconvulsive therapy sessions during the first lockdown. As outpatient ECT's (Electroconvulsive therapy) were stopped during the first lockdown, one client maintaining well on maintenance ECT relapsed. The client had to be hospitalized and restarted on ECT. Another client (with the diagnosis of chronic schizophrenia and bouts of violent behavior) bit a staff member due to relapse of symptoms and needed to be hospitalized. Both clients were transported to the hospital with RFS vehicle and driver. The first author, who is part of the RFS team, visited the residential facilities 2-3 times a week and administered injections personally, in addition to managing psychiatric emergencies. Wherever necessary, additional medications were used. All consultations except emergencies were online during 
the pandemic, and the situation was better during the second lockdown.

The staff had worries that they would get infected. They were appreciated and compensated for work done during the lockdown. As the professional staff worked in close proximity to clients, they were given PPE (personal protective equipment) kits when they were working. The support staff were provided gloves.

During the first and second wave lockdown, the support staff (like cook and attenders) were requested to stay at the facility instead of traveling back to their homes to ensure safety for the clients. It also ensured no interruption in the facility's routine and provided food to residents and staff on time. The staff were provided accommodation at premises for a month. Every time, they returned RT-PCR for COVID-19 was done.

As the first wave ebbed by September 2020, clients settled into a routine with the group and other rehabilitation activities until the second wave started.

\section{Handling COVID-19 Outbreak in the Residential Facilities}

COVID-19 cases were reported in Asha half-way home during the first wave, and affected clients were hospitalized after informing families.

During the second wave, 4 of the clients from Jyothi long-stay home were COVID-19 positive. The acute shortage of hospital beds during the second wave posed additional challenges. The clients were isolated immediately after the positive test result. The two clients with reduced oxygen saturation were hospitalized after some effort. The hospitalized clients were worried and anxious as they were alone without access to family members. The psychotropic medication dosage for hospitalized patients was titrated in liaison by the treating psychiatrist.

Two others (one tested positive, and the second client had active symptoms but a negative report) could not be hospitalized, and there were no local family members. They were isolated on the first floor of the building under the supervision of a nurse to stop the further spread of the virus and give proper care for those tested positive. The nurse was trained to handle clients' mental health concerns by a professional staff under our supervision. A cleaning staff quarantined with the client accepted management's request to help as outside help was impossible. Handling the emotions of clients during their quarantine period was challenging. The staff made sure to be regularly in touch with the clients over the telephone, addressing their anxieties. It was stressful for the staff to be on call for long hours. All the four clients who were quarantined recovered fully within two weeks' time.

During the outbreak, all clients and staff underwent COVID-19 tests. The disclosure of the outbreak to clients and staff was handled sensitively. The families of clients with COVID negative results and those from within the city were requested to take their wards home for a short period up until the end of lockdown. This had to be done due to a shortage of staff during this period. Only clients whose families were overseas stayed in the residential facility.

The management was in constant touch with the clients, staff, and families for advice, guidance, and reassurance, which helped handle the issues sensitively.

\section{Concerns Related to the Staff Team}

The Chetana staff worked from home during lockdowns. The staff was paid salary in full during this period. The management provided monetary compensation for the staff who put in more work during the lockdown and quarantine with additional days of leave benefits.

During the second wave of COVID-19, staff from Asha half-way home (2 support and one professional staff) and Jyothi long-stay home (two professional staff) contracted COVID-19. One staff and his family had to be hospitalized. Other 3 staff were isolated in a single room with attached bathroom for quarantine in RFS premises. All staff recovered in 2 weeks time. The management took care of all medical treatment and expenses. As the first and second authors were continuously in touch with the staff and the treating physician, the staff morale was high. Their colleagues worked additional hours to take care of the clients. The management extended complete financial support for COVID-19 treatment of staff without a cut in their salaries.

\section{Role of Families}

Families of clients were in constant touch with the clients over video and audio calls during the 
pandemic. They were very appreciative of the efforts put in by the management and the staff team to care for their wards. None accused the management as to why some clients got infected. The families knew that they would have had a much more difficult time caring for the clients at home.

One client who was hospitalized for COVID-19 had a private attender who also tested positive. The concerned family took care of medical treatment and quarantine needs of the private attender also. The staff contacted the family of clients hospitalized for COVID-19 twice a day on how the patient was doing. Families felt RFS cared for their health and welfare.

Families donated generously to the COVID-19 treatment fund for the staff. Periodic updates were shared on WhatsApp groups. Some arranged for sanitizers, N95 masks, gloves, thermometers, and oximeters for staff and clients. Some families offered their private vehicles to transport clients to the hospital for the COVID-19 test. Before the second wave, families staying in Bangalore took their wards back home until the lockdown got lifted as requested.

Cooperation and camaraderie with families are the hallmarks of RFS.

\section{COVID-19 Vaccination Drive}

Clients in the residential facilities above 45 years of age and all the staff members were double vaccinated between February and May 2021 with Covishield Vaccine. The Chetana daycare centre staff made inquiries regarding the vaccination status of clients and their families. Under the Government's special scheme for people with disabilities, the management organized a camp to get all the clients and families vaccinated. Staff helped some families who needed help to book appointments online. There was a deep sense of appreciation and relief from the satisfied family members.

\section{Shift to Online Meetings and Virtual Platforms}

Even before the pandemic, the staff were communicating with clients and their families by WhatsApp groups. During the pandemic, finding the right time to contact the chetana clients family was a challenge as some family members were working from home. The family members were enthusiastic and felt cared for. Online conversations were easier for clients with mental illness than clients with intellectual disability. As comprehension of clients with intellectual disability was limited, instructions had to be repeated.

During the second wave lockdown, Chetana engaged clients online (through WhatsApp video calls, Zoom, and Google Meet) in various topic discussions chosen as per wishes of clients and families. The clients were encouraged to share their individual concerns during online sessions and they were assigned home work to make sure they continued their routines.

The annual Jyothi family meet was held virtually instead of in person, and families across the globe joined for the program.

The clients in residential facilities availed teleconsultations from their doctors unless there was a compelling indication for an in-person assessment.

Meetings and orientation programs for students, which earlier used to happen in person, were discontinued to avoid the risk of COVID-19 transmission through visitors. Student internships have been started on virtual mode from June 2021 after the second lockdown was lifted.

\section{Managing Finances}

As an NGO, the facilities are run with funds generated from fees paid by clients, sales of products made by clients, fees collected from interns, and sporadic donations.

Though the organization continued to incur expenses for staff and infrastructure during the pandemic, regular sources of income reduced considerably. As Chetana was closed for a substantial duration during the pandemic, most clients did not pay fees, but despite that, the staff members were paid their full salary. With the looming prospect of a third wave, client patronage for Chetana and the financial situation is a matter of great concern.

The finances of residential facilities are better positioned as the residents continue to pay for services availed. The management offered concessions to Asha clients for the period they stayed at home during the pandemic.

As no visitors were allowed to enter RFS premises due to COVID-19 concerns, there was no sale of 
products made by clients and no revenue from student internships. The pandemic has strained the organization's finances, and the management has dipped into the reserves accumulated over the years to sustain services.

\section{Conclusion}

The COVID-19 pandemic and lockdowns stretched RFS resources to their fullest. Since Chetana had to close for prolonged periods, the usual fee collection had to be abandoned! The freedom of clients in residential facilities was curtailed, and they were not permitted to go out of the facility to reduce the risk of virus exposure. This robbed them of the opportunity to shop, eat out and watch movies in the theatre, which was part of their weekly routine, all such activities they were used to and enjoyed over long periods. During the lockdown period, the support staff ( cook and attenders) were asked to stay back at our premises, as there was no provision for them to come to work from home regularly. Managing the COVID-19 outbreak in the residential facilities was not only difficult but also demanding. The management had to take the unprecedented step of asking local clients to go home due to staff shortage. Despite the adverse financial situation, RFS has upheld its ethos of caring for clients, treating all stakeholders fairly, and ensuring that the staff morale was kept high.

Funding No funding.

\section{Declarations}

Conflict of interest The authors declare that they have no competing interests.

\section{References}

1. COVID Live Update. $193,422,846$ Cases and 4,151,689 deaths from the coronavirus-worldometer. 2021. https:// www.worldometers.info/coronavirus/.

2. Golechha M. COVID-19, India, lockdown and psychosocial challenges: What next? Int $J$ Soc Psychiatry. 2020;66(8):830-2. https://doi.org/10.1177/002076402 0935922 .
3. Hamada K, Fan X. The impact of COVID-19 on individuals living with serious mental illness. Schizophr Res. 2020;222:3-5. https://doi.org/10.1016/j.schres.2020.05. 054.

4. Javed A, Afzal M. COVID-19 and the management of chronic mental illnesses. J Psychosoc Rehabil Ment Health. 2020;7(3):207-8. https://doi.org/10.1007/s40737-02000188-3.

5. Jayarajan D, Sivakumar T, Torous JB, Thirthalli J. Telerehabilitation in Psychiatry. Indian $\mathrm{J}$ Psychol Med. 2020;42(5_suppl):57S-62S. https://doi.org/10.1177/ 0253717620963202.

6. Kola L, Kohrt BA, Hanlon C, Naslund JA, Sikander S, Balaji M, Benjet C, Cheung EYL, Eaton J, Gonsalves P, Hailemariam M, Luitel NP, Machado DB, Misganaw E, Omigbodun O, Roberts T, Salisbury TT, Shidhaye R, Sunkel C, et al. COVID-19 mental health impact and responses in low-income and middle-income countries: reimagining global mental health. Lancet Psychiatry. 2021;8(6):535-50. https://doi.org/10.1016/S2215-0366(21)00025-0.

7. Kopelovich SL, Monroe-DeVita M, Buck BE, Brenner C, Moser L, Jarskog LF, Harker S, Chwastiak LA. Community mental health care delivery during the COVID-19 pandemic: practical strategies for improving care for people with serious mental illness. Community Ment Health J. 2021;57(3):405-15. https://doi.org/10.1007/s10597-02000662-z.

8. Parry SJ, Chamorro V, Mohan R. Responding to COVID-19 in psychiatric rehabilitation: collaboration is vital. J Psychosoc Rehabil Ment Health. 2020;7(3):209-10. https://doi. org/10.1007/s40737-020-00180-x.

9. Ravindran S, Channaveerachari NK, Seshadri SP, Kasi S, Manikappa SK, Cherian AV, Sudhir P, Govindan R, Reddy B, Christopher AD, George S. Crossing barriers: Role of a tele-outreach program addressing psychosocial needs in the midst of COVID-19 pandemic. Asian J Psychiatry. 2020;53:102351. https://doi.org/10.1016/j.ajp.2020. 102351.

10. Roy A, Jayarajan D, Sivakumar T. Income generation programs for persons with mental health challenges: practices from 13 Indian Mental Health Rehabilitation Centers. Indian J Psychol Med. 2020. https://doi.org/10.1177/ 0253717620959759.

11. Sundaram SK, Kumar S. Tracing the development of psychosocial rehabilitation from its origin to the current with emphasis on the Indian context. Indian $\mathrm{J}$ Psychiatry. 2018;60(6):253. https://doi.org/10.4103/psychiatry. IndianJPsychiatry_437_17.

12. Tazaki M. Impact of COVID-19 on people with mental disabilities in Japan. J Psychosoc Rehabil Ment Health. 2020;7(2):113-4. https://doi.org/10.1007/s40737-02000173-w.

Publisher's Note Springer Nature remains neutral with regard to jurisdictional claims in published maps and institutional affiliations. 